\title{
Nitrogen Diffusion in Amorphous Silicon Nitride Isotope Multilayers Probed by Neutron Reflectometry
}

\author{
H. Schmidt \\ AG Thermochemie und Mikrokinetik, Fakultät für Natur-und Materialwissenschaften, \\ TU Clausthal, D-38678 Clausthal-Zellerfeld, Germany \\ M. Gupta \\ Laboratory for Neutron Scattering, ETH Zürich \& PSI, Paul Scherrer Institute, Villigen, CH-5232, Switzerland \\ M. Bruns \\ Institut für Instrumentelle Analytik, Forschungszentrum Karlsruhe GmbH, D-76021 Karlsruhe, Germany \\ (Received 24 October 2005; published 7 February 2006)

\begin{abstract}
Amorphous silicon nitride is a model system for a covalently bound amorphous solid with a low atomic mobility where reasonable values of self-diffusivities are still lacking. We used neutron reflectometry on isotope enriched $\mathrm{Si}_{3}{ }^{14} \mathrm{~N}_{4} / \mathrm{Si}_{3}{ }^{15} \mathrm{~N}_{4}$ multilayers to determine nitrogen self-diffusivities ranging from $10^{-24}$ to $10^{-21} \mathrm{~m}^{2} / \mathrm{s}$ between 950 and $1250^{\circ} \mathrm{C}$. Time dependent diffusivities observed at $1150^{\circ} \mathrm{C}$ indicate the presence of structural relaxation. For long annealing times (relaxed state) the diffusivities follow an Arrhenius law with an activation enthalpy of $(3.6 \pm 0.4) \mathrm{eV}$. The results are indicative of a direct diffusion mechanism without the involvement of thermal point defects.
\end{abstract}

DOI: 10.1103/PhysRevLett.96.055901

PACS numbers: 66.30.Hs, 61.12.Ha, 61.43.Dq

Amorphous solids differ from their crystalline counterparts by the absence of long range order and are metastable in the sense that they may transform into the crystalline state by heating the material at elevated temperatures. The thermal stability, the stability against environmental impact, and structural relaxations of noncrystalline solids are essentially influenced by self-diffusion of the elemental constituents. Extensive studies on self-diffusion were carried out in recent years on oxide glasses [1,2] and metallic glasses [3]. Up to now, however, no systematic studies are available for nonoxide covalently bound amorphous solids, like silicon, silicon carbide, and silicon nitride [4]. This is due to the fact that it is extremely difficult to perform successful experiments with conventional methods on such materials. Commonly, diffusion experiments are carried out by measuring the modification of a tracer distribution (radioactive or stable isotopes) after annealing at elevated temperatures by sputter sectioning or secondary ion mass spectrometry (SIMS) [5]. Thus, tracer isotopes are deposited by ion implantation, by isotope exchange from the gas phase, or by deposition methods like chemical vapor deposition or pulsed laser deposition. However, for the essential constituents of nonmetallic amorphous solids no suitable radioactive tracers are available for the performance of extensive measurements, because of a too short half-life period of radioactive $\mathrm{Si}, \mathrm{B}, \mathrm{C}, \mathrm{O}$, and $\mathrm{N}$ isotopes. This fact prevents or at least extremely complicates the use of the standard radiotracer technique, which is the state-of-the-art method in diffusivity determination in metals and intermetallics due to its high detection sensitivity. To circumvent this problem, it is necessary to use rare stable isotopes whose natural abundances reduce the respective dynamic ranges of the tracers. In covalently bound solids, self-diffusivities are very low due to the high binding energy of the components in the order of 3-4 eV. Thus, diffusion experiments have to be carried out with high resolution depth profiling methods like SIMS, where diffusion lengths down to $5 \mathrm{~nm}$ can be measured. However, such diffusion lengths are often still too large for covalent amorphous materials, which start to crystallize before any measurable diffusion occurs.

In order to perform self-diffusion measurements on a covalently bound amorphous solid we choose amorphous silicon nitride $\left(\mathrm{Si}_{3} \mathrm{~N}_{4}\right)$ as a model system. Thin films of silicon nitride exhibit a variety of attractive properties, like high hardness, chemical stability, a high dissociation temperature, high thermal shock resistance, and a wide band gap. They are used in mechanical, optical, and electronic applications in various branches of technology (for a review see, e.g., [6]). In this work, diffusion measurements were carried out on isotopically enriched $\mathrm{Si}_{3}{ }^{14} \mathrm{~N}_{4} / \mathrm{Si}_{3}{ }^{15} \mathrm{~N}_{4}$ multilayers, which were produced by magnetron sputter deposition. Because of the different coherent neutron scattering lengths of ${ }^{14} \mathrm{~N}(9.37 \mathrm{fm})$ and ${ }^{15} \mathrm{~N}(6.44 \mathrm{fm})$, an ideal contrast for neutron scattering techniques is given. Using neutron reflectometry, Bragg peaks occur due to the periodically modulated structure of the multilayer. Selfdiffusivities are achieved by measuring the decay of the first and second order Bragg peaks after annealing at elevated temperatures. The superior resolution of this technique allows the determination of diffusion lengths well below $1 \mathrm{~nm}$.

Homogeneous amorphous $\mathrm{Si}_{3} \mathrm{~N}_{4}$ layers were produced by reactive rf magnetron sputter deposition using a mixture 
of $50 \mathrm{vol} \%$ argon and $50 \mathrm{vol} \%$ nitrogen in combination with a silicon target. As substrates, single crystalline $\mathrm{Si}$ wafers and also polished polycrystalline $\mathrm{SiC}$ disks were used. The elemental composition of the sputtered layers was measured by non-Rutherford backscattering spectroscopy to be stoichiometric $\mathrm{Si}_{3} \mathrm{~N}_{4}$ [7]. The O impurity concentration was determined to be less than 0.2 at. \%, and the hydrogen content was measured to be about 0.2 at. $\%$ by additional nuclear reaction analysis. Structural characterization of the layers was carried out by $\mathrm{x}$-ray diffractometry and Fourier transform infrared spectrometry both confirming the amorphous state of the material. First traces of crystallization (about 5 at. \% of crystallized phase) were detected after annealing the layers for $10 \mathrm{~h}$ at $1300^{\circ} \mathrm{C}$ [7]. For the diffusion measurements, isotopically enriched multilayers of the form $\left[\mathrm{Si}_{3}{ }^{14} \mathrm{~N}_{4}(19 \mathrm{~nm}) / \mathrm{Si}_{3}{ }^{15} \mathrm{~N}_{4}(6 \mathrm{~nm})\right]_{20}, \quad\left[\mathrm{Si}_{3}{ }^{14} \mathrm{~N}_{4}(22 \mathrm{~nm}) /\right.$ $\left.\mathrm{Si}_{3}{ }^{15} \mathrm{~N}_{4}(7 \mathrm{~nm})\right]_{20}$, and $\left[\mathrm{Si}_{3}{ }^{14} \mathrm{~N}_{4}(17 \mathrm{~nm}) /\right.$ $\left.\mathrm{Si}_{3}{ }^{15} \mathrm{~N}_{4}(4 \mathrm{~nm})\right]_{20}$ were produced using either nitrogen with natural isotope ratio $\left({ }^{14} \mathrm{~N} /{ }^{15} \mathrm{~N}=99.633 \% / 0.366 \%\right)$ or isotopically enriched nitrogen $\left({ }^{14} \mathrm{~N} /{ }^{15} \mathrm{~N}=0.5 \% /\right.$ 99.5\%). The layer thickness was determined by SIMS (CAMECA IMS $3 \mathrm{~F}, 15 \mathrm{kV}, 40 \mathrm{nA}, \mathrm{O}^{-}$). Diffusion annealing was carried out in nitrogen (980 mbar) in the temperature range between 950 and $1250{ }^{\circ} \mathrm{C}$. Determination of the nitrogen self-diffusivities was obtained by neutron reflectivity in the time-of-flight mode of the AMOR reflectometer at the Swiss spallation neutron source (SINQ), located at the Paul Scherrer Institute (Switzerland) [8]. At incoming neutron wavelengths, $\lambda$, between 0.2 and $0.9 \mathrm{~nm}$ the reflectivity patterns were measured at three different incident angles at $\theta=0.3^{\circ}$, $0.7^{\circ}$, and $1.3^{\circ}$.

Figure 1 compares the neutron reflectivity of a uniform $\mathrm{Si}_{3} \mathrm{~N}_{4}$ layer that is not isotopically enriched and of an isotopic $\left[\mathrm{Si}_{3}{ }^{14} \mathrm{~N}_{4}(19 \mathrm{~nm}) / \mathrm{Si}_{3}{ }^{15} \mathrm{~N}_{4}(6 \mathrm{~nm})\right]_{20}$ multilayer

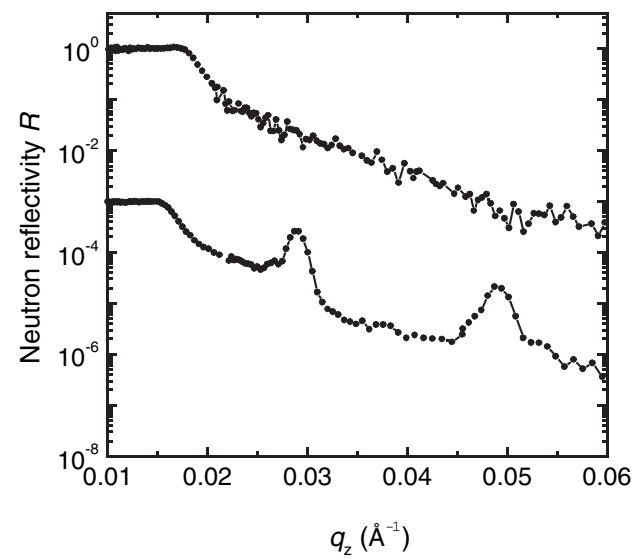

FIG. 1. Neutron reflectivity pattern of a $\left[\mathrm{Si}_{3}{ }^{14} \mathrm{~N}_{4}(19 \mathrm{~nm}) /\right.$ $\left.\mathrm{Si}_{3}{ }^{15} \mathrm{~N}_{4}(6 \mathrm{~nm})\right]_{20}$ isotopic multilayer (bottom) compared to the pattern of a not enriched $\mathrm{Si}_{3} \mathrm{~N}_{4}$ film (top). The reflectivity pattern of the multilayer is divided by a factor of 1000 for clarity. in the as-deposited state. For the multilayer, first and second order Bragg peaks occur at scattering vectors $q_{z}=$ $4 \pi \sin \theta / \lambda=0.029$ and $0.049 \AA^{-1}$ due to the periodic variation of the neutron scattering length of the two isotopes. For the uniform layer no such structures occur, and only the background resulting from Fresnel reflectivity is visible.

In Fig. 2 the neutron reflectivity patterns of a multilayer after diffusion annealing for $168 \mathrm{~h}$ at $1000{ }^{\circ} \mathrm{C}$ is shown in comparison to an as-deposited multilayer. The patterns are multiplied by $q_{z}^{4}$ in order to correct for the background. A decrease of the intensity of both Bragg peaks is clearly visible for the annealed sample. The self-diffusivities can be calculated according to the expression $[9,10]$

$$
I(t)=I_{0} \exp \left(-\frac{8 \pi^{2} n^{2} D}{d^{2}} t\right)
$$

where $I_{0}$ is the intensity of the $n$th order Bragg peak at time $t=0, D$ is the diffusivity, and $d$ is the bilayer periodicity. Diffusivities were derived from the intensities of the first and second order Bragg peaks and averaged. The results are shown in Fig. 3, where the nitrogen self-diffusivities in amorphous silicon nitride are plotted as a function of reciprocal temperature between 950 and $1250{ }^{\circ} \mathrm{C}$. The diffusivities range between $10^{-24}$ and $10^{-21} \mathrm{~m}^{2} / \mathrm{s}$, which corresponds to diffusion lengths of $l=(2 D t)^{1 / 2}$ between 1 and $4 \mathrm{~nm}$ for annealing times between 0.67 and $200 \mathrm{~h}$. The diffusivities obtained for isotope multilayers on $\mathrm{Si}$ and $\mathrm{SiC}$ substrates, respectively, show no significant differences within error limits, which discards a possible influence of the substrate on diffusion. The temperature dependence of the diffusivities can be described by an Arrhenius equation

$$
D=D_{0} \exp \left(-\frac{\Delta H^{D}}{k_{\mathrm{B}} T}\right)
$$

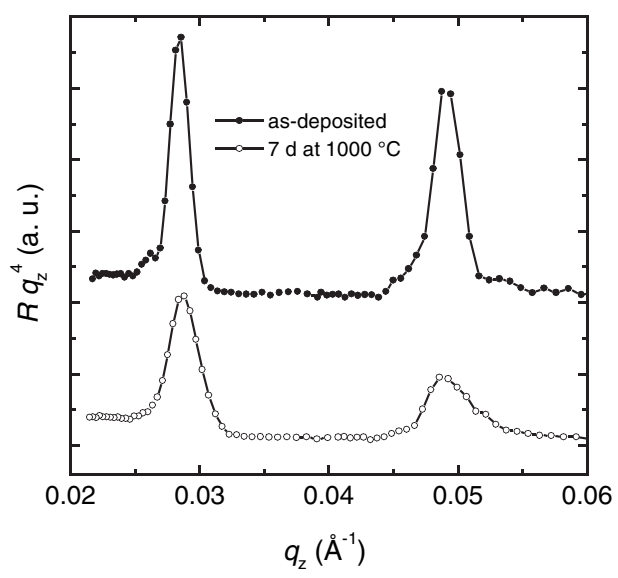

FIG. 2. Neutron reflectivity pattern of a $\left[\mathrm{Si}_{3}{ }^{14} \mathrm{~N}_{4}(19 \mathrm{~nm}) /\right.$ $\left.\mathrm{Si}_{3}{ }^{15} \mathrm{~N}_{4}(6 \mathrm{~nm})\right]_{20}$ isotopic multilayer in the as-deposited state (shifted for clarity) and after annealing for $7 d$ at $1000{ }^{\circ} \mathrm{C}$. The patterns are multiplied by $q_{z}^{4}$ in order to correct for the background. 


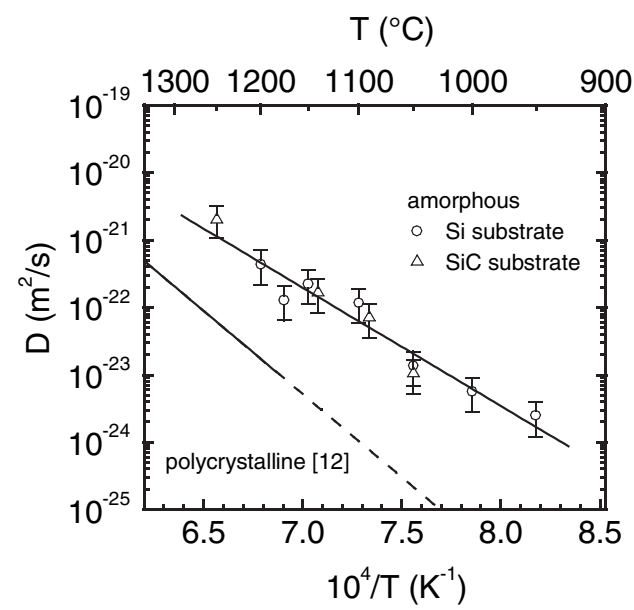

FIG. 3. Nitrogen diffusivities in amorphous $\mathrm{Si}_{3} \mathrm{~N}_{4}$ films as a function of reciprocal temperature. The dashed line is an extrapolation of the experimental data on polycrystalline $\mathrm{Si}_{3} \mathrm{~N}_{4}$ films represented by the solid line (after Ref. [12]).

with an activation enthalpy of $\Delta H^{D}=(3.6 \pm 0.4) \mathrm{eV}$ and a preexponential factor of $D_{0}=1 \times 10^{-9} \mathrm{~m}^{2} / \mathrm{s}$ (error: $\log _{10} D_{0}= \pm 1.3$ ), where $T$ is the annealing temperature and $k_{\mathrm{B}}$ is the Boltzmann constant.

Under the assumption that the diffusion process is similar to a conventional point-defect mechanism, the activation enthalpy of diffusion can be considered as the sum of an enthalpy of formation and an enthalpy of migration of the diffusing species (vacancies, interstitials, complexes). The formation enthalpy of a nitrogen vacancy is calculated to be between 3.1 and $6.5 \mathrm{eV}$ (in crystalline $\mathrm{Si}_{3} \mathrm{~N}_{4}$ ), depending on the exact chemical composition (N-rich or Sirich limit) [11]. These values are larger than or close to the lower limit of the experimentally measured activation enthalpy of diffusion. Thus, it appears unlikely that diffusion takes place via thermally equilibrated point defects. In contrast, a direct mechanism without the mediation of these defects is more plausible where the diffusing atoms can jump into a vacant neighboring site. Such vacant sites can be identified with excess free volume, which is formed during deposition with magnetron sputtering. Consequently, the activation enthalpy of diffusion is essentially determined by the migration enthalpy alone.

Also shown in Fig. 3 are nitrogen diffusivities in polycrystalline $\mathrm{Si}_{3} \mathrm{~N}_{4}$ as determined by SIMS [12]. They exhibit a higher activation enthalpy of $(4.9 \pm 0.4) \mathrm{eV}$ and also a higher preexponential factor of $1 \times 10^{-6} \mathrm{~m}^{2} / \mathrm{s}$ leading to about 1 to 2 orders of magnitude lower diffusivities in the temperature range investigated. In contrast to amorphous silicon nitride, an activation enthalpy in this range is more compatible with a diffusion mechanism mediated by thermally activated point-defect motion [12].

In Fig. 4 nitrogen diffusivities are shown that were determined for annealing times of 1,4 , and $14.5 \mathrm{~h}$ at $1150^{\circ} \mathrm{C}$. The diffusivities decrease by about 1 order of

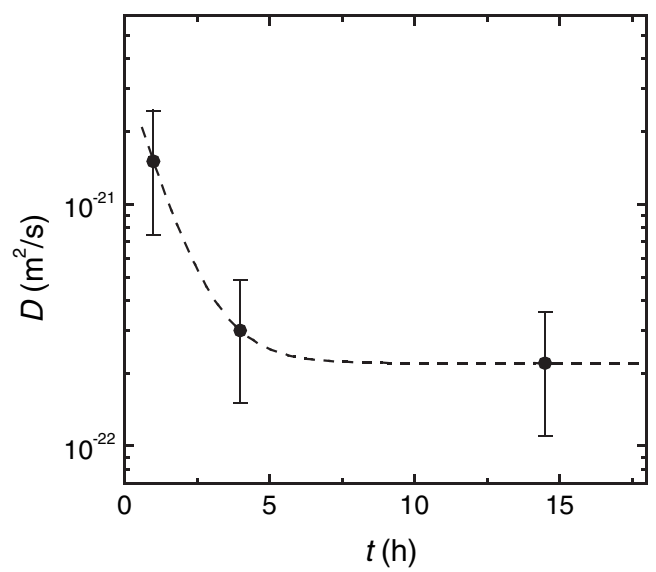

FIG. 4. Self-diffusivities of nitrogen in amorphous $\mathrm{Si}_{3} \mathrm{~N}_{4}$ for various annealing times at $1150{ }^{\circ} \mathrm{C}$. The dashed line is a guide to the eye.

magnitude with increasing annealing time. This indicates that structural relaxation plays a considerable role, where a metastable amorphous state is transformed into a more stable but still amorphous state. During this process, free volume and consequently the number of defects responsible for diffusion may decrease with annealing time due to defect annihilation. As a result, diffusivities decrease also. After annealing for about $4 \mathrm{~h}$, a relaxed amorphous state is reached, where further annealing does not alter diffusivities. Similar effects are also observed, e.g., for metallic glasses [3]. The diffusivities plotted in Fig. 3 are all derived for long annealing times, so that they correspond to the well relaxed state.

A comparison of the present results to other types of materials is given in Fig. 5 where the activation enthalpy of

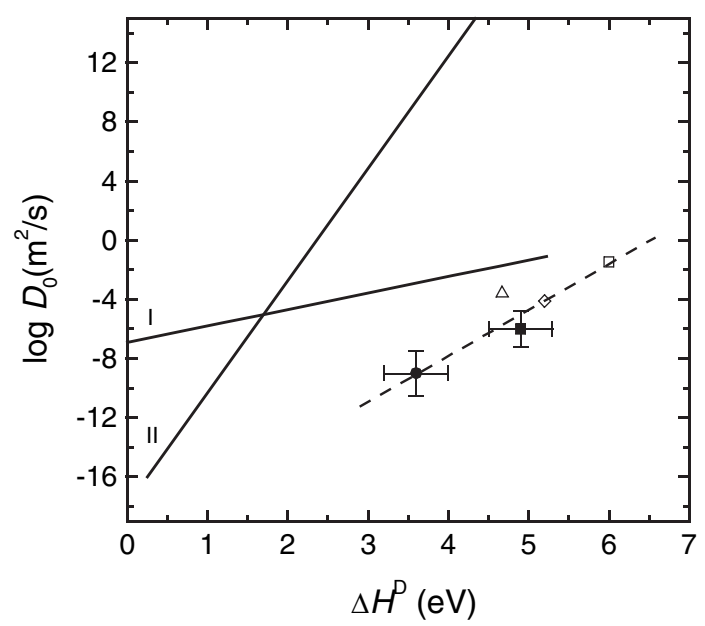

FIG. 5. Plot of $\Delta H^{D}$ vs $\log D_{0}$ for the self-diffusion in amorphous and crystalline solids: $\mathrm{N}$ in amorphous $(\mathbf{O})$ (this work) and polycrystalline (ם) [12] magnetron sputtered $\mathrm{Si}_{3} \mathrm{~N}_{4}, \mathrm{O}$ in CVD derived vitreous $\mathrm{SiO}_{2}(\triangle)$ [19], $\mathrm{Si}$ in thermally grown $\mathrm{SiO}_{2}(\diamond)$ [17], and in fused $\mathrm{SiO}_{2}(\square)$ [18]. Also shown are crystalline metals (line I) [3] and bulk metallic glasses (line II) [3]. 
diffusion is plotted versus the logarithm of the preexponential factor. Literature data of crystalline metals [3] show a correlation between $\Delta H^{D}$ and $D_{0}$ (straight line I), which is often interpreted as an indication for a common or similar diffusion mechanism. For crystalline metals a diffusion mechanism with localized point defects (mainly vacancies) is operating. Also included in line I are recent data for typical single crystalline semiconductors like $\mathrm{Si}$ [13], Ge [14], and GaAs [15], which show no significant deviation from that line within an estimated error range of $\log D_{0}= \pm 1$. For semiconductors, diffusion via point defects that are extended over several neighboring lattice sites is assumed [16]. Completely different are the data for metallic glasses (line II), where a typical collective diffusion mechanism is observed [3]. Here, several atoms participate in the jump process and very high values of $D_{0}$ occur for $\Delta H^{D}$ values between 2 and $5 \mathrm{eV}$. As obvious, the present results on amorphous silicon nitride are in accordance with none of these two lines. A several orders of magnitude lower preexponential factor occurs, if compared for the same activation enthalpy. Also given in Fig. 5 are data for the diffusion of $\mathrm{Si}[17,18]$ and $\mathrm{O}$ [19] in glassy $\mathrm{SiO}_{2}$ (for an overview see Refs. [2,20]) where also considerably lower preexponential factors are found. A key structure element of amorphous $\mathrm{Si}_{3} \mathrm{~N}_{4}$ is tetrahedrally coordinated silicon, where the nitrogen atoms are connected to a three-dimensional amorphous network structure [21]. For glassy $\mathrm{SiO}_{2}$ the basic structure element is a $\mathrm{SiO}_{4}$ tetrahedron, which is quite similar to a $\mathrm{SiN}_{4}$ tetrahedron, however, with a more ionic bonding. As given in Fig. 5 by a dashed line, the data of amorphous $\mathrm{Si}_{3} \mathrm{~N}_{4}$ and glassy $\mathrm{SiO}_{2}$ also indicate a correlation between $\Delta H^{D}$ and $D_{0}$. According to these observations, short-range ordered tetrahedra seem to play a fundamental role in the description of atomic motion in these materials. However, this does not necessarily mean that $\mathrm{SiN}_{4}$ tetrahedra are the diffusing species. $\mathrm{SiN}_{4}$ tetrahedra can be regarded as structural building blocks (like $\mathrm{SiO}_{4}$ tetrahedra), which are characteristic for diffusional motion due to the specific short-range order. As diffusing species, nitrogen atoms and also molecular fragments $\mathrm{SiN}_{x}$ $(x=1$ to 4$)$ are possible.

In conclusion, we used neutron reflectometry on isotope enriched $\mathrm{Si}_{3}{ }^{14} \mathrm{~N}_{4} / \mathrm{Si}_{3}{ }^{15} \mathrm{~N}_{4}$ multilayers to determine selfdiffusivities in a covalently bound amorphous solid. Very low diffusivities $\left(10^{-24}-10^{-21} \mathrm{~m}^{2} / \mathrm{s}\right)$ are found between 950 and $1250{ }^{\circ} \mathrm{C}$, which follow an Arrhenius law with an activation enthalpy of $3.6 \mathrm{eV}$ in the relaxed state. The results are indicative of a direct diffusion mechanism without thermal point defects. In a correlation plot (logarithm of the preexponential factor versus activation enthalpy) the data of $\mathrm{Si}_{3} \mathrm{~N}_{4}$ considerably deviate from those of crystalline metals and semiconductors and also from those of metallic glasses, while they are very close to those of glassy $\mathrm{SiO}_{2}$. This suggests a diffusion mechanism where tetrahedral structural units play a key role.
The authors thank U. Geckle for thin film preparation and M. Rudolphi and H. Baumann for carrying out the n-RBS, NRRA, and FTIR measurements. Thanks also to G. Borchardt for fruitful discussions. This work is based on experiments performed at the Swiss spallation neutron source SINQ, Paul Scherrer Institute, Villigen, Switzerland. This research project has been supported by the European Commission under the 6th Framework Programme through the Key Action: Strengthening the European Research Area, Research Infrastructures, Contract No. RII3-CT-2003-505925.

[1] G. H. Frischat, Ionic Diffusion in Oxide Glasses (Trans Tech, Aedermannsdorf, 1975).

[2] M. A. Lamkin, F. L. Riley, and R. J. Fordham, J. Eur. Ceram. Soc. 10, 347 (1992).

[3] F. Faupel, W. Frank, M. P. Macht, H. Mehrer, V. Naundorf, K. Rätzke, H. R. Schober, S. K. Sharma, and H. Teichler, Rev. Mod. Phys. 75, 237 (2003).

[4] Diffusion in Non-metallic Solids, edited by D. L. Beke, Landolt-Börnstein NS III, Vol. 33 (Springer, Berlin, 1999).

[5] Diffusion in Solid Metals and Alloys, edited by H. Mehrer, Landolt-Börnstein NS III, Vol. 26 (Springer, Berlin, 1990).

[6] F. L. Riley, J. Am. Ceram. Soc. 83, 245 (2000).

[7] H. Schmidt, W. Gruber, G. Borchardt, M. Bruns, M. Rudolphi, and H. Baumann, Thin Solid Films 450, 346 (2004).

[8] D. Clemens, P. Gross, P. Keller, N. Schlumpf, and M. Könnecke, Physica (Amsterdam) 276B, 140 (2000).

[9] T. Mizoguchi and M. Murata, Jpn. J. Appl. Phys. 30, 1818 (1991).

[10] J. Speakmann, P. Rose, J. A. Hunt, N. Cowlam, R. E. Somekh, and A.L. Greer, J. Magn. Magn. Mater. 156, 411 (1996).

[11] I. Tanaka, K. Tatsumi, M. Nakano, H. Adachi, and F. Oba, J. Am. Ceram. Soc. 85, 68 (2002).

[12] H. Schmidt, G. Borchardt, M. Rudolphi, H. Baumann, and M. Bruns, Appl. Phys. Lett. 85, 582 (2004).

[13] H. Bracht, E. E. Haller, and R. Clark-Phelps, Phys. Rev. Lett. 81, 393 (1998).

[14] H. D. Fuchs et al., Phys. Rev. B 51, 16817 (1995).

[15] L. Wang, L. Hsu, E. E. Haller, J. W. Erickson, A. Fischer, K. Eberl, and M. Cardona, Phys. Rev. Lett. 76, 2342 (1996).

[16] W. Frank, U. M. Gösele, H. Mehrer, and A. Seeger, in Diffusion in Crystalline Solids, edited by G. E. Murch and A. S. Nowick (Academic Press, Orlando, 1984), p. 63.

[17] G. Brebec, R. Seguin, C. Sella, J. Bevenot, and J.C. Martin, Acta Metall. 28, 327 (1980).

[18] T. Takahashi, S. Fukatsu, K. M. Itoh, M. Uematsu, A. Fujiwara, H. Kageshima, Y. Takahashi, and K. Shirishi, J. Appl. Phys. 93, 3674 (2003).

[19] J. C. Mikkelsen, Appl. Phys. Lett. 45, 1187 (1984).

[20] H. Bracht, R. Staskunaite, E. E. Haller, P. Fielitz, G. Borchardt, and D. Grambole, J. Appl. Phys. 97, 046107 (2005).

[21] J. F. Justo and C. R. S. da Silva, Diffus. Defect Data, Pt. A 206, 19 (2002). 